\title{
Influence of benzene exposure, fat content, and their interactions on erythroid-related hematologic parameters in petrochemical workers: a cross- sectional study
}

\author{
Xue Zhang \\ Sun Yat-Sen University \\ Qifei Deng \\ Sun Yat-Sen University \\ Zhini He \\ Southern Medical Univesity \\ Jie Li
}

Sun Yat-Sen University

\section{Xiaoju Ma}

Sun Yat-Sen University

\section{Zhaorui Zhang}

Sun Yat-Sen University

Dehua Wu

Sun Yat-Sen University

\section{Xiumei Xing}

Sun Yat-Sen University

Jing Peng

Sun Yat-Sen University

Hongyu Guo

Sun Yat-Sen University

\section{Ming Huang}

Guangdong province hospital for occupational disease prevention and treatment

\section{Liping Chen}

Sun Yat-Sen University

\section{Shanfeng Dang}

Occupational disease prevention and treatment institute of Sinopec Maoming petrochemical company Yanqun Zhu

Occupational disease prevention and treatment institute of Sinopec Maoming petrochemical company Zhengbao Zhang

Southern medical university 


\section{Boyi Yang}

Sun Yat-Sen University

\section{Hailan Wang}

Guangdong province hospital for occupational disease prevention and treatment

\section{Wen Chen}

Sun Yat-Sen University

\section{Yongmei Xiao ( $\nabla$ xiaoym@mail.sysu.edu.cn )}

Sun Yat-Sen University

\section{Research article}

Keywords: benzene exposure, fat content, interactions, erythroid-related hematologic parameters

Posted Date: March 2nd, 2020

DOI: https://doi.org/10.21203/rs.2.16880/v2

License: (c) (1) This work is licensed under a Creative Commons Attribution 4.0 International License.

Read Full License

Version of Record: A version of this preprint was published at BMC Public Health on March 23rd, 2020. See the published version at https://doi.org/10.1186/s12889-020-08493-z. 


\section{Abstract}

Background: Ubiquitously distributed benzene is a known hematotoxin. Increasing evidence has suggested that erythroid-related hematologic parameters may be sensitive to benzene exposure. Fat content, which is also closely associated with erythroid-related hematologic parameters, may affect the distribution and/or metabolism of benzene, and eventually benzene-induced toxicity. Methods: To explore the influence of benzene exposure, fat content, and their interactions on erythroid-related hematologic parameters, we recruited 1669 petrochemical workers and measured their urinary S-phenylmercapturic acid (SPMA) concentration and erythroid-related hematological parameters. Indices for fat content included body fat percentage (BF\%), plasma total cholesterol (TC) and triglycerides (TG) , and occurrence of fatty liver. Results: The dose-response curve revealed U-shaped nonlinear relationships of SPMA with hematocrit $(\mathrm{HCT})$ and mean corpuscular hemoglobin concentration $(\mathrm{MCHC})$ ( $\mathrm{P}$-overall $<0.001$, and $\mathrm{P}$ nonlinear $<0.015$ ), as well as positive linear associations and $r$-shaped nonlinear relationships of continuous fat content indices with erythroid-related hematological parameters ( $P$-overall $\leq 0.005$ ). We also observed modification effects of fat content on the associations between benzene exposure and erythroid-related hematological parameters, with workers of lower or higher $\mathrm{BF} \%$ and TG more sensitive to benzene-induced elevation of MCHC ( $P$ interaction $=0.021)$ and benzene-induced decrease of HCT ( $P$ interaction $=0.050)$, respectively. We also found that some erythroid-related hematologic parameters differed between subgroups of workers with different SPMA levels and fat content combination. Conclusions: Our study suggested that benzene exposure, fat content, and their interactions may affect erythroid-related hematological parameters in petrochemical workers in a complex manner that are worthy of further investigation.

\section{Background}

Benzene is a representative single-ring aromatic compound which ubiquitously distributes in our environment due to its high volatility and extensive emission sources. Benzene occurs naturally in crude oil and gas emission from volcanoes or forest fires. However, most environmental contamination and human exposure results from man-made sources like the emissions from motor vehicles, cigarette smoke, and the production and usage of petroleum products and common consumer goods, such as adhesives, paints, rubber products, solvents, and pharmaceutical products [1]. With the increasing development of industrialization and globalization, many developing countries have achieved great growth in the production of petrochemical products. This expands the population of petrochemical workers potentially exposed to benzene [2]. Furthermore, occupational exposures are likely to occur at higher concentrations than those encountered in the general environment.

Benzene is a serious public health concern and has been classified as a known human carcinogen [3]. After entering the body mostly through inhalation, benzene is metabolically activated by cytochrome P450 enzymes, and its metabolites distributes to lipid-rich tissues (including bone marrow) according to their fat content and rate of perfusion by blood, resulting in multiple adverse health effects. Hematotoxicity is the most widely-studied health effect for benzene exposure [4]. Multiple studies have 
linked benzene exposure with the abnormality of hematologic parameters, such as the reduction in the counts of white blood cell (WBC), red blood cell (RBC), neutrophil, and lymphocyte, even at low exposure levels ( $<1 \mathrm{ppm}$ ) [5-7]. A decreased WBC count has been considered as a key clinical sign of benzeneinduced hematotoxicity [8]. However, increasing evidence supports that erythrocytes may be more sensitive at low benzene exposure levels. A large-scale study conducted in Korean workers revealed that RBC count was the only hematologic parameter that was reduced by low-level benzene exposure $(<1$ $\mathrm{ppm}$ ) in male workers [9]. Our previous studies had also shown that RBC count was decreased in C57BL/6J male mice treated with $1 \mathrm{ppm}$ benzene, and that erythroid cell differentiation of hematopoietic progenitor cells was inhibited by exposure to low-dose hydroquinone (a toxic metabolite of benzene) $[10,11]$. However, the literature investigating the effects of benzene exposure on erythroid-related hematologic parameters contains inconsistent findings, something needing resolution through the conduct of more epidemiologic studies.

As benzene mainly accumulates in the fat-enriched tissues upon entry into human body [12], fat content in the body and in tissues or organs (such as blood and liver) might affect the distribution and/or metabolism of benzene, and may eventually affect benzene-induced toxicity. Moreover, studies have demonstrated that fat content may also have an effect on erythroid-related hematologic parameters. The percentage of erythroid precursors of bone marrow cells was significantly increased in rats fed with a fatty-rich diet [13]. High-fat diet induced dysfunction of erythrocytes by increasing their oxidative stress levels and reducing their survival in a rat model of high fat diet-induced obesity [14]. Also, patients with non-alcoholic fatty liver disease were more likely to have a high red cell distribution width (RDW) and elevated hemoglobin $(\mathrm{Hb})$ levels $[15,16]$. In addition, indicators for fat content in blood, such as serum cholesterol (TC) and triglycerides (TG), were also associated with hematological diseases [17]. However, few studies have investigated the effects of fat content on the associations of benzene exposure with erythroid-related hematologic parameters.

Thus, in order to investigate the influence of benzene exposure, fat content in the body, in blood, and in liver, and their interactions on erythroid-related hematologic parameters, we performed a cross-sectional study among 1669 petrochemical workers. We quantified their benzene exposure levels by measuring a widely-used internal exposure marker, S-phenylmercapturic acid (SPMA), estimated their body fat percentage (BF\%), measured TC and TG in blood, evaluated the occurrence of fatty liver by ultrasound, and measured multiple commonly-used erythroid-related hematologic parameters. We used restricted cubic spline (RCS) functions to characterize the dose-response associations of benzene exposure and fat content with erythroid-related hematologic parameters. We further probed into their interaction effects on erythroid-related hematologic parameters. The present study might further enhance our knowledge about the determinants of the severity of benzene induced-erythroid hematotoxicity.

\section{Methods}

\subsection{Study design and setting}


In order to investigate the influence of benzene exposure, fat content, and their interactions on erythroidrelated hematologic parameters, we conducted a cross-sectional study in workers from two state-run petrochemical companies which are located in Guangzhou and Maoming in Guangdong Province, China.

\subsection{Study participants}

We firstly recruited a total of 1802 workers and then intentionally excluded: a) workers who had been working for less than one year in their petrochemical plants; b) workers with self-reported and/or diagnosed carcinomas, hematological diseases, and/or immune diseases; $c$ ) workers taking any medicine in the preceding two weeks; d) workers experiencing X-ray examination for any reason in the preceding 1 month; and e) workers unwilling to provide biological samples or doing so in insufficient volume. Eventually, a total of 1669 workers were recruited in this study.

After participants signed informed consent, we administered a structured questionnaire to collect their information on demographic characteristics, lifestyle (such as smoking and drinking habits), medical history, and occupational experience (such as working years and workplaces). Participants who had smoked $\geq 1$ cigarette/day for $\geq 1$ year were considered as smokers, and those who drank wine and/or other alcoholic beverages at least once a week for $\geq 1$ year were classified as drinkers. Workers that had been working in workshops such as petroleum refining, chemical production, and petroleum processing were considered as benzene-exposed group, while administration staffs were considered as non-exposed subjects.

After the interview, we collected $\sim 20 \mathrm{~mL}$ morning urine sample and $\sim 2 \mathrm{~mL}$ ethylenediaminetetraacetic acid (EDTA)-anticoagulated venous blood sample from each participant after overnight fasting. The present study had received ethical approval from the Ethical Review Committee at School of Public Health, Sun Yat-Sen University.

\subsection{Measurement of urinary SPMA concentration}

Urinary SPMA concentration was determined with liquid chromatography/electrospray tandem mass spectrometry (LC-MS/MS) described in detail previously [18]. In brief, $5 \mathrm{~mL}$ urine sample for each participant was centrifuged at $800 \mathrm{~g}$ for $5 \mathrm{~min}$. An aliquot of $500 \mu \mathrm{L}$ supernatant was mixed with $500 \mu \mathrm{L}$ of $10 \mathrm{mM}$ sodium acetate buffer $(\mathrm{pH}=6.3)$, and treated with $50 \mu \mathrm{L}$ of $10 \mu \mathrm{g} / \mathrm{L}$ SPMA-d5 working solution. After solid-phase extraction (Waters Oasis ${ }^{\circledR}$ MAX), the samples were analyzed with LC-MS/MS (Agilent, US). SPMA (purity $98 \%$ ) is used for qualitative analysis, and standard curves were drawn for quantitative analysis. SPMA-d5 working solution as internal standard was used for quality control. The inter-assay coefficient of variation (CV) (10 different sample preparations, 10 measurements at different days by different persons) was $10.21 \%$. The detection limit for urinary SPMA was $0.01 \mu \mathrm{g} / \mathrm{L}$ and the concentrations of samples with levels below detection limit were substituted to $0.005 \mu \mathrm{g} / \mathrm{L}$. SPMA concentration was standardized by urinary creatinine and expressed as $\mu \mathrm{g} / \mathrm{g}$ creatinine.

\subsection{Measurement of fat content indices}


We measured the height of our participants using a wall-mounted stadiometer. The participants stood upright on a firm surface, looking straight ahead, arms at sides, with shoes removed and feet together. Their shoulders, buttocks, and heels were required to be touching the wall. We also measured the weight for participants with a calibrated standing balance scale. Participants removed shoes, heavy outer clothing, and items from pockets. Height and weight were measured twice for each participant to ensure accurate measurement. The average height and weight were used to calculate body mass index (BMI) according to the following formula:

$\mathrm{BMI}=$ Weight $(\mathrm{kg}) /$ Height $(\mathrm{m})^{2}$

Then, we calculated $\mathrm{BF} \%$ for each participant according to the following formula [19]:

$\mathrm{BF} \%=(1.20 \times \mathrm{BMI})+(0.23 \times \mathrm{Age})-10.8 \times$ Gender -5.4

In this formula, "Gender" =1 for male workers, while "Gender" =0 for female workers.

Plasma samples extracted from EDTA-anticoagulated blood were used for the quantitative analysis of TC and TG by automatic biochemical analyzer (Cobas, Switzerland). The inter-assay CVs for TC and TG (10 different sample preparations, 10 measurements at different days by different persons) were $8.62 \%$ and $12.97 \%$, respectively. Two professional sonographers separately detected the occurrence of fatty liver disease for each participant by abdominal ultrasound examination based on clinical diagnostic criteria of fatty liver [20], and the concordance rate was 100\%.

\subsection{Measurement of erythroid-related hematologic parameters}

In the present study, we used automatic hematology analyzer (Sysmex, Japan) to measure seven commonly-used erythroid-related hematologic parameters in EDTA-anticoagulated venous blood samples, including RBC count $\left(\times 10^{12} / \mathrm{L}\right)$, Hb concentration $(\mathrm{g} / \mathrm{L})$, hematocrit $(\mathrm{HCT})(\%)$, mean corpuscular volume (MCV) ( $\mathrm{fL})$, mean corpuscular hemoglobin concentration $(\mathrm{MCHC})(\mathrm{g} / \mathrm{L})$, RDW-coefficient of variation (RDW-CV) (\%), and RDW-standard deviation (RDW-SD) ( $\mathrm{fL}$ ). These hematologic parameters were measured within 2 hours after sample collection, and each blood sample was assayed in duplicate. The average levels for these erythroid-related hematologic parameters were included in the following statistical analyses.

\subsection{Statistical analyses}

We examined the distributions of all numerical variables by Kolmogorov-Smirnov tests. As the urinary SPMA concentration had a left-skewed distribution, it was normalized by natural logarithm (In) transformation before statistical analyses [21]. Several variables were adjusted as confounders in our statistical analyses, including age (continuous), gender (male/female), smoking status (smokers/nonsmokers), drinking status (drinkers/non-drinkers), working years (continuous), and workplace (exposed/control group). As BF\% was calculated based on age and gender, we didn't adjust age and/or gender in all following analyses involving BF\%. 
We evaluated the differences of general characteristics between the exposed workers and controls with Student's $t$-test for continuous variables and chi-square test for categorical variables. We analyzed the between-group differences of SPMA concentration, fat content indices, and erythroid-related hematologic parameters by multivariate analysis of covariance, with adjustment for the above-mentioned confounding variables except for workplace.

RCS models, which have been widely-used to represent nonlinear relationships for continuous independent variables, were used to characterize patterns of changes in erythroid-related hematologic parameters with the changes in continuous exposure (including SPMA, BF\%, TC, and TG) in the total population, while adjusting for the above-mentioned covariates. Continuous exposure was included into RCS models with 4 default knots located at the 5th, 35th, 65th, and 95th percentiles. The reference values on RCS curves were set at the median values for continuous exposure, and the values on $\mathrm{Y}$ axis represented the differences in erythroid-related hematologic parameters between individuals with any value of continuous exposure with those with median levels. The outputted $P$-overall indicate $P$ values for test of overall association, and $P$-nonlinear indicate $P$ values for test of nonlinear association. We used covariate-adjusted generalized linear models (GLMs) to analyze the effects of occurrence of fatty liver disease ( $\mathrm{no}=0$, and yes $=1$ ) on erythroid-related hematologic parameters (as dependent variables) in the total population, and those of SPMA and all fat content indices with dependent variables in workers with different general characteristics. We further explored the modification effects of general characteristics on these associations by adding an interaction term of independent variable (continuous) and stratified variable (categorical) in GLMs.

Then, we divided our workers into three subgroups $(\mathrm{T} 1, \mathrm{~T} 2$, and $\mathrm{T} 3)$ according to the tertiles of $\mathrm{BF} \%, \mathrm{TC}$, and TG, respectively, and divided workers into two subgroups according to the occurrence of fatty liver (No/Yes). We analyzed the associations of SPMA with erythroid-related hematologic parameters in subgroups with different fat content indices by covariate-adjusted GLMs, and explored the modification effects of fat content indices on these associations by adding an interaction term of SPMA (continuous) and fat content categories (categorical) in GLMs. In order to evaluate the combined effects of benzene exposure and fat content, we also divided our participants into four subgroups based on the median SPMA concentration (low/high) and the median continuous fat content indices or the occurrence of fatty liver. Workers with low SPMA and with low BF\%, TC, TG, or without fatty liver were defined as the reference groups, and the differences of erythroid-related hematologic parameters between other subgroups and controls were evaluated with covariate-adjusted GLMs.

RCS analyses were performed by rms package in R software (Version 3.5.2), and other statistical analyses were performed using SPSS (Version 22.0). Two-tailed $P<0.05$ was considered statistically significant.

\section{Results}

\subsection{Subject characteristics}


The general characteristics, benzene exposure levels, fat content indices, and erythroid-related hematologic parameters among workers in different workplaces were presented in Table 1. Significant differences were observed in terms of all general characteristics between controls and exposed workers (all $P<0.05$ ). Median SPMA levels in the exposed workers were about 2.1 -fold higher than that in control subjects $(P<0.001)$. BF\% and MCHC were significantly lower while HCT, MCV, and RDW-SD were higher in exposed workers than control subjects (all $P<0.05$ ).

\subsection{Associations of SPMA and fat content indices with erythroid-related hematologic parameters}

We conducted RCS analyses to characterize the dose-response relationships of SPMA and continuous fat content indices with erythroid-related hematologic parameters (Figure 1-4). We observed that SPMA had inverse U-shape and U-shape nonlinear relationships with HCT and $\mathrm{MCHC}$, respectively $(P$-overall $<0.001$, and $P$-nonlinear < 0.015) (Figure 1). When In-transformed SPMA was lower than about -1.8, HCT increased and MCHC decreased with the increase of SPMA. However, when In-transformed SPMA was higher than about -1.8, HCT decreased and MCHC increased with an increase of SPMA.

As for continuous fat content indices, the dose-response curves revealed positive linear associations of $\mathrm{BF} \%$ with RBC count, $\mathrm{Hb}$, and $\mathrm{HCT}$, of TC with RDW-SD, and of TG with $\mathrm{MCHC}(P$-overall $\leq 0.005$, and $P$ nonlinear > 0.05) (Figure 2-4). Additionally, both TC and TG had r-shape nonlinear relationships with $\mathrm{Hb}$ and HCT $(P$-overall $<0.001$, and $P$-nonlinear $<0.02)$. TG also had r-shape nonlinear dose-response relationship with RBC count $(P$-overall $<0.001$, and $P$-nonlinear $=0.002) . \mathrm{Hb}$ and $\mathrm{HCT}$ increased until around $6 \mathrm{mmol} / \mathrm{L}$ of TC and then slightly declined. RBC count, Hb, and HCT increased until around 1.25 $\mathrm{mmol} / \mathrm{L}$ of $\mathrm{TG}$ and then levels off (Figure 2-4).

Similarly, workers with fatty liver disease had relatively higher RBC count, $\mathrm{Hb}$, and $\mathrm{HCT}\left(P<5.0 \times 10^{-5}\right)$ (Table 2). These results, taken together with those in Figure 1-4, suggest that benzene exposure and fat content might have complex effects on erythroid-related hematologic parameters.

In addition, we also investigated these associations in workers with different characteristics (including gender, smoking status, and/or drinking status) with GLMs (See Table S1-S5, Additional file 1). These results indicated that general characteristics could modify the associations of SPMA and fat content with some erythroid-related hematologic parameters, with such influence more pronounced in females, nonsmokers, and non-drinkers $\left(P_{\text {interaction }}<0.05\right)$.

\subsection{Interactions and combined effects of SPMA and fat content on erythroid-related hematologic parameters}

To investigate whether benzene exposure and fat content have interaction effects on erythroid-related hematologic parameters, we analyzed the modification effects of fat content indices on the associations between urinary SPMA levels and erythroid-related hematological parameters. We found that both T1 and T3 subgroups of $\mathrm{BF} \%$ had a more pronounced positive association of SPMA with MCHC, while the association was weaker and insignificant in workers of T2 group $\left(P_{\text {interaction }}=0.021\right)$. Similarly, we also 
observed that both T1 and T3 subgroups of TG also had a more pronounced negative association of SPMA with HCT, while the association was also weaker and insignificant in workers of T2 group $\left(P_{\text {interaction }}=0.050\right)$ (Figure 5 and Table S6, Additional file 1). Associations of SPMA with other erythroidrelated hematological parameters were not significantly different among workers with different fat content levels $\left(P_{\text {interaction }}>0.050\right)$ (Table S6).

We also investigated the combined effects of benzene exposure with fat content on erythroid-related hematologic parameters. We divided our participants into four subgroups according to their median level of SPMA and median levels of continuous fat content indices or the occurrence of fatty liver, with workers of "Low SPMA + Low fat indices or without fatty liver" as the reference groups (Table 3). The general observation is that RBC count, $\mathrm{Hb}$, and $\mathrm{HCT}$ was significantly decreased in workers with higher $\mathrm{BF} \%$, and significantly elevated in workers with higher TC and TG and with fatty liver, regardless of SPMA levels (between-group $P<0.05$ ). MCV was also significantly elevated in workers with higher TC, once again without respect to SPMA levels (between-group $P<0.05$ ). MCHC and RDW-SD values did not significantly differ between the four subgroups (between-group $P>0.05$ ). RDW-CV, however, was slightly, but consistently elevated in workers with higher SPMA and lower BF\%, TC, TG, or without fatty liver, and decreased in workers with higher SPMA and higher TC and TG or with fatty liver (between-group $P<0.05$ ).

\section{Discussion}

In the present cross-sectional study conducted in 1669 petrochemical workers, we found that benzene exposure and fat content in body, in blood, and in liver might affect erythroid-related hematologic parameters in linear, U-shaped, or r-shaped dose-response patterns. Furthermore, we found that $\mathrm{BF} \%$ and TG might modify the associations of benzene exposure with erythroid-related hematologic parameters. Specifically, workers of low or high BF\% were more sensitive to benzene-induced elevation of $\mathrm{MCHC}$ than their median BF\% counterparts, and workers of low or high TG were more sensitive to benzene-induced decrease of HCT than their median TG counterparts. We also found that most erythroid-related hematologic parameters differed among subgroups with varying combinations of SPMA levels and fat content, indicating there were combined effects between benzene exposure and fat content on erythroidrelated hematologic parameters.

The possible mechanisms underlying the benzene-mediated hematoxicity has been widely-studied in recent years [22]. Toxic benzene metabolites (such as hydroquinone, phenol, and catechol) produced by metabolism in the liver and bone marrow act on hematopoietic stem progenitor cells and mature blood cells, suppress the function of hematopoietic tissue, and cause abnormality in blood cell counts. Increasing evidence found that the erythrocyte is also sensitive to benzene exposure and even low-dose ( $<1 \mathrm{ppm}$ ) benzene can disturb red blood cells $[10,11]$. Thus, we focused on the associations of benzene with erythroid-related hematologic parameters in the present study. Although substantial previous studies have explored the linear relationship between benzene exposure and erythroid-related hematologic parameters $[9,23]$, their findings were inconsistent and few studies have further investigated whether there were any non-linear relationships. Since RCS allows easy visualization and flexible associations 
between an exposure and outcome, we applied this model to fully examine the dose-response relationships.

In the present study, urinary SPMA, a sensitive and specific benzene exposure biomarker which is rarely influenced by other factors (such as diet) [24], was found to have inverse U-shape and U-shape nonlinear relationships with HCT and MCHC, respectively, with the inflection points of In-transformed SPMA at around -1.8 (equivalent to about $0.17 \mu \mathrm{g} / \mathrm{g}$ creatinine of SPMA). HCT is the proportion of whole blood that is made up of red blood cells, and may be used to check for conditions such as anemia, dehydration, malnutrition, and leukemia. $\mathrm{MCHC}$ is the mean $\mathrm{Hb}$ concentration in a specific volume of red blood cells, and is calculated by dividing $\mathrm{Hb}$ by HCT. An increase in MCHC indicates autoimmune hemolytic anemia or hereditary spherocytosis, and a decrease indicates a thalassemia or an anemia due to iron deficiency. In the present study, the association of SPMA with MCHC was in the direction opposite to that of SPMA with HCT, namely with the increase of SPMA, HCT increased first and then decreased, while MCHC decreased first and then increased. This suggests that the relationship between benzene exposure and some erythroid-related hematologic parameters may be discrepant at different exposure levels. MCHC was found to be inversely associated with blood benzene in residents living near petrochemical areas at environmental exposure concentration [25], which was consistent with our finding that at lower benzene exposure levels, MCHC decreased with increasing SPMA. Furthermore, significantly decreased HCT and increased $\mathrm{MCHC}$ were observed in individuals working in high traffic and industrial areas with high concentration of benzene [26], which was also consistent with our finding that at higher benzene exposure levels, MCHC increased and HCT decreased with increasing SPMA. However, a study conducted in children observed negative correlation between HCT and benzene at environmental exposure levels [27], which is contrary to our result that HCT increased with increasing SPMA at lower exposure levels. This discrepancy might be explained by age-related differences in susceptibility to benzene, as toxicokinetic processes that determine dose differ between children and adults, not to mention vulnerability of the hematologic system to toxic insult. Taken together, our findings provided a new insight that benzene exposure could influence erythroid-related hematologic system in a nonlinear pattern which might be dependent on benzene exposure levels. Further studies are needed to clarify the exposure conditions under which the variable response of each erythroid-related hematologic parameter might occur.

Fat content is involved in metabolism and body energy reserves. Under-load or over-load of fat content may cause adverse health effects. Low fat content often reflects poor nutrition status and may increase susceptibility to diseases related to environmental pollutant exposure [28]. As for high fat content, it may cause inflammation and disturb blood cell counts [29], and is also an important risk factor for cardiovascular diseases and metabolic disorders [30]. In the present study, we used BF\%, TC, TG, and the occurrence of fatty liver disease to represent fat content in body, in blood, and in liver. The reason for using $\mathrm{BF} \%$ instead of $\mathrm{BMI}$ to represent the body fat content was that $\mathrm{BF} \%$ is a better predictor for body fat mass based on age and gender $[19,31]$. Studies have shown that some erythroid-related hematologic parameters, such as RBC count, $\mathrm{Hb}$, and $\mathrm{HCT}$, were significantly positively correlated with waist to hip ratio and waist circumference $[32,33]$. In the present study, we also observed that $\mathrm{BF} \%, \mathrm{TC}$, and $\mathrm{TG}$ are 
significantly positively associated with erythroid-related hematologic parameters in linear or r-shaped dose-response patterns, and workers with fatty liver disease had higher RBC count, $\mathrm{Hg}$ concentration, and $\mathrm{HCT} \%$ than their non-diseased counterparts. The underlying mechanisms of the effects of fat content on erythroid-related hematologic parameters may hinge upon membranes of erythrocytes, which are rich in polyunsaturated fatty acids, being very sensitive to changes in fat content in vivo [34]. Excessive fat content may cause abnormal counts and function of RBCs by affecting their membrane composition, fluidity, and rheology [32].

We also conducted stratification analyses to understand the effect of general characteristics on the associations of benzene and fat content with erythroid-related hematologic parameters. Our findings indicated that most of these associations were more remarkable in females, in non-smokers, and in nondrinkers. Physiological differences between males and females may account for variable toxicological responses to poisons [35]. Studies have shown that females have a higher percentage of body fat, gender alters the biotransformation of benzene, and women may be more susceptible to blood alterations caused by benzene exposure [36]. Cigarette smoking is one of the most important sources of benzene, and studies have demonstrated that smoking may affect the associations of benzene exposure with blood cell counts [23]. In addition, smoking and drinking have been found to stimulate hematopoietic system, and result in the changes of hematologic parameters, such as RBC count, $\mathrm{Hb}$, and MCV $[37,38]$. Our findings and those of others suggest that more attention should be paid to the influence of gender and lifestyle factors (such as smoking and drinking) when surveilling the health of populations occupationally exposed to benzene.

Moreover, fat content might affect the distribution and/or metabolism of benzene, and eventually affect benzene-induced toxicity. However, few studies have investigated the interaction effects of benzene exposure with fat content on erythroid-related hematologic parameters. In the present study, we observed that the associations of SPMA with MCHC and HCT were significantly influenced by BF\% and TG, respectively, with workers at the lower and higher extremes of each fat content index more sensitive to benzene-induced elevation of MCHC and decrease of HCT. These interesting findings indicated that lower and higher fat content may be risk factors for benzene-induced hematotoxicity. For example, a lower fat content might cause lower energic reserves and affect immunological function, impairing the ability to cope with environmental stressors [39]. On the other hand, chronic benzene exposure decreased white blood cell numbers, but only in rats that had a large volume of fat tissue [40]. Furthermore, we also found that most erythroid-related hematologic parameters differed among subgroups of workers having different combinations of benzene exposure and fat content (i.e., low or high BF\%, TC, or TG and with or without fatty liver). As benzene mainly accumulates in lipid-rich tissue due to its lipophilicity [41], high fat content may allow for more benzene to accumulate in the body, slow the elimination of benzene and its active metabolites, and thus enhance the toxicity of benzene. Additionally, benzene exposure and high fat content can both induce excessive reactive oxygen species which might cause erythrocytic dysfunction and cell apoptosis, ultimately making RBCs more easily absorbed by spleen and inducing abnormal blood cell counts $[42,43]$. Benzene exposure can also increase the production of proinflammatory cytokine [26], and obese individuals are considered to be in a low-grade inflammatory state [44]. Studies 
have indicated that cytokines and inflammatory environment might both interfere with the RBC maturation in bone marrow [45]. Moreover, some enzymes and polymorphisms in their encoding genes may affect both benzene-induced hematotoxicity and fat content levels. For example, polymorphisms in myeloperoxidase (MPO) (such as $463 \mathrm{G}>\mathrm{A}$ ) and $\mathrm{NAD}(\mathrm{P}) \mathrm{H}$ dehydrogenase quinone 1 (NQ01) (such as C609T) may alter enzyme activity, which is associated with TC levels [46,47], TG levels [47], and the risk of benzene poisoning [48]. These enzymes and their genetic polymorphisms might be the underlying mechanisms of the interaction effects between benzene exposure and fat content on erythroid-related hematologic parameters. Although further studies are strongly needed to confirm our findings and explore underlying mechanisms for our observed hematologic changes, the present study strongly suggest fat content might influence the health status of benzene-exposed individuals. It remains less clear, however, under what circumstances a lower or higher fat content would increase one's sensitivity to benzeneinduced toxicity or prove protective.

Our study had some major strengths. Firstly, we used multiple fat content indictors to represent the fat content in body, in blood, and in liver. Furthermore, the present study was the first to examine the interaction effects of benzene exposure with fat content on erythroid-related hematologic parameters. Our findings may have important implications for the health surveillance of environmental and occupational benzene-exposed population alike, though considerable uncertainty exits as to whether our findings are extrapolatable to the general population. Finally, our subjects may be co-exposed to other hazards which might also influence erythroid-related hematologic parameters. Hence, further studies are needed to validate our findings, explore the potential mechanisms, and evaluate the effects of co-exposure to other hazards on our findings.

\section{Conclusions}

In summary, the present study revealed benzene exposure and fat content in body, in blood, and in liver might all affect erythroid-related hematologic parameters. We also observed that fat content might affect the severity of benzene-induced erythroid-related hematotoxicity, with workers of lower or higher fat content more sensitive to benzene-induced erythroid-related hematotoxicity. We also found there might be combined effects between benzene exposure and fat content. Our findings may have some implications in health surveillance and prevention of benzene-induced erythroid-related hematotoxicity for environmental- and occupational-exposed populations. However, further studies should be performed to validate our current findings and explore their potential mechanisms.

\section{List Of Abbreviations}

WBC, white blood cell; RBC, red blood cell; RDW, red cell distribution width; Hb, hemoglobin; TC, total cholesterol; TG, triglycerides; SPMA, S-phenylmercapturic acid; BF\%, body fat percentage; RCS, restricted cubic spline; EDTA, ethylenediaminetetraacetic acid; LC-MS/MS, liquid chromatography/electrospray tandem mass spectrometry; CV, coefficient of variation; BMI, body mass index; HCT, hematocrit; MCV, mean corpuscular volume; $\mathrm{MCHC}$, mean corpuscular hemoglobin concentration; RDW-CV, red cell 
distribution width-coefficient of variation; RDW-SD, red cell distribution width-standard deviation; Intransformation, natural logarithm transformation; $P$-overall, $P$ values for test of overall association; $P$ nonlinear, $P$ values for test of nonlinear association; GLMs, generalized linear models; $P_{\text {interaction }} P$ values for test of interaction; $P_{\text {trend }}, P$ values for test of linear association, MPO, myeloperoxidase; NQ01, $\mathrm{NAD}(\mathrm{P}) \mathrm{H}$ dehydrogenase quinone 1.

\section{Declarations}

Ethics approval and consent to participate: The present study had received ethical approval from the Ethical Review Committee at School of Public Health, Sun Yat-Sen University. Participation of our study subjects did not occur until after informed consent was obtained.

Consent for publication: Not applicable.

Availability of data and materials: The datasets used and/or analyzed during the current study are available from the corresponding author on reasonable request.

Competing interests: The authors declare that they have no competing interests.

Funding: This research was supported by the key Program of the National Natural Science Foundation of China (81430079), regular National Natural Science Foundation of China (81372962, 81402658, 81273099, and 81602877), the National "Twelfth Five-Year Plan" for Science \& Technology (2014BAI12B01), Guangdong Province Doctor Staring Program Grant (2016A030310163), and the Young Teachers Training Program of Sun Yat-sen University (Grant number 18ykpy14). The funding body had no role in the design of the study, collection, analyses and interpretation of data nor in writing the manuscript.

Authors' contributions: $X Z$ and QD wrote the draft paper; $Q D, L C, B Y, W C$, and $Y X$ revised the manuscript and improved the language; $X Z, Q D, Z H, Z Z, D W$, and $B Y$ analyzed the data; $W C, Y X, Q D, X Z, Z H, J L, X X$, and $L C$ designed the study; $X Z, Q D, J L, X M, J P, H G$, and $Y X$ contributed to data collection; $M H, S D, Y Z, Z Z$, $\mathrm{HW}, \mathrm{WC}$, and $\mathrm{YX}$ contributed to the progress of the study; and all authors have approved the final article.

Acknowledgements: We thank all study participants, research staffs, and students who participated in this work.

\section{References}

1. Zhang X, Xue Z, Li H, Yan L, Yang Y, Wang Y, et al. Ambient volatile organic compounds pollution in China. J Environ Sci-China. 2017;55:69-75.

2. Heibati B, Pollitt KJG, Karimi A, Yazdani Charati J, Ducatman A, Shokrzadeh M, et al. BTEX exposure assessment and quantitative risk assessment among petroleum product distributors. Ecotox Environ Safe. 2017;144:445-449. 
3. Cumulative Index to IARC monographs on the evaluation of the carcinogenic risk of chemicals to humans. IARC Monogr Eval Carcinog Risk Chem Hum. 1986;39:379-403.

4. Poynter JN, Richardson M, Roesler M, Blair CK, Hirsch B, Nguyen P, et al. Chemical exposures and risk of acute myeloid leukemia and myelodysplastic syndromes in a population-based study. Int $\mathrm{J}$ Cancer. 2017;140(1):23-33.

5. Lan Q, Zhang L, Li G, Vermeulen R, Weinberg RS, Dosemeci M, et al. Hematotoxicity in Workers Exposed to Low Levels of Benzene. Science. 2004;306(5702):1774-1776.

6. Casale T, Sacco C, Ricci S, Loreti B, Pacchiarotti A, Cupelli V, et al. Workers exposed to low levels of benzene present in urban air: Assessment of peripheral blood count variations. Chemosphere. 2016;152:392-398.

7. Rothman N, Li GL, Dosemeci M, Bechtold WE, Marti GE, Wang YZ, et al. Hematotoxicity among Chinese workers heavily exposed to benzene. Am J Ind Med. 1996;29(3):236-246.

8. Cliona M. McHale LZMT. Current understanding of the mechanism of benzene-induced leukemia in humans: implications for risk assessment. Carcinogenesis. 2012;2(33):240-252.

9. Koh $D$, Jeon $H$, Lee $S$, Ryu $H$. The relationship between low-level benzene exposure and blood cell counts in Korean workers. Occup Environ Med. 2015;72(6):421-427.

10. Liang B, Chen Y, Yuan W, Qin F, Zhang Q, Deng N, et al. Down-regulation of miRNA-451a and miRNA486-5p involved in benzene-induced inhibition on erythroid cell differentiation in vitro and in vivo. Arch Toxicol. 2018;92(1):259-272.

11. Yuan W, Sun Q, Jiang Y, Zhang X, Chen L, Xie C, et al. MiR-146a affects the alteration in myeloid differentiation induced by hydroquinone in human CD34(+) hematopoietic progenitor cells and HL-60 cells. Toxicol Res-UK. 2016;5(3):848-858.

12. Peyret T, Krishnan K. QSARs for PBPK modelling of environmental contaminants. Sar Qsar Environ Res. 2011;22(1-2):129-169.

13. Krosniak M, Papiez MA, Kaczmarczyk J, Francik R, Panza MG, Covelli V, et al. Influence of fructose and fatty-rich diet combined with vanadium on bone marrow cells. Biol Trace Elem Res. 2013;155(2):276-282.

14. Chang T, Liu K, Chang CS, Su C, Chen S, Lee Y, et al. Ferric Citrate Supplementation Reduces RedBlood-Cell Aggregation and Improves CD163+ Macrophage-Mediated Hemoglobin Metabolism in a Rat Model of High-Fat-Diet-Induced Obesity. Mol Nutr Food Res. 2018;62(2):1700442.

15. Yang $W$, Huang $H$, Wang $Y, Y u X$, Yang Z. High red blood cell distribution width is closely associated with nonalcoholic fatty liver disease. Eur J Gastroen Hepat. 2014;26(2):174-178.

16. Giorgio V, Mosca A, Alterio A, Alisi A, Grieco A, Nobili V, et al. Elevated Hemoglobin Level Is Associated With Advanced Fibrosis in Pediatric Nonalcoholic Fatty Liver Disease. J Pediatr Gastr Nutr. 2017;65(2):150-155.

17. Kiss A, Telek B, Rak K. Hypocholesterolemia and other lipoprotein disorders in myelofibrosis. In Orv Hetil. 1994;135(48):2643-6. 
18. Schettgen T, Musiol A, Alt A, Kraus T. Fast determination of urinary S-phenylmercapturic acid (SPMA) and S-benzylmercapturic acid (S-BMA) by column-switching liquid chromatography-tandem mass spectrometry. J Chromatogr B. 2008;863(2):283-292.

19. Deurenberg P, Weststrate JA, Seidell JC. Body mass index as a measure of body fatness: age- and sex-specific prediction formulas. Brit J Nutr. 1991;65(2):105-114.

20. Albhaisi S, Issa D, Alkhouri N. Non-alcoholic fatty liver disease: a pandemic disease with multisystem burden. Hepatobiliary Surg Nutr. 2018;7(5):389-391.

21. Hernan MA, Hernandez-Diaz S, Werler MM, Mitchell AA. Causal knowledge as a prerequisite for confounding evaluation: an application to birth defects epidemiology. Am J Epidemiol. 2002;155(2):176-184.

22. Smith MT. Advances in Understanding Benzene Health Effects and Susceptibility. Annu Rev Publ Health. 2010;31(1):133-148.

23. Doherty BT, Kwok RK, Curry MD, Ekenga C, Chambers D, Sandler DP, et al. Associations between blood BTEXS concentrations and hematologic parameters among adult residents of the U.S. Gulf States. Environ Res. 2017;156:579-587.

24. Hoet P, De Smedt E, Ferrari M, Imbriani M, Maestri L, Negri S, et al. Evaluation of urinary biomarkers of exposure to benzene: correlation with blood benzene and influence of confounding factors. Int Arch Occ Env Hea. 2009;82(8):985-995.

25. Chen Q, Sun H, Zhang J, Xu Y, Ding Z. The hematologic effects of BTEX exposure among elderly residents in Nanjing: a cross-sectional study. Environ Sci Pollut R. 2019;26(11):10552-10561.

26. Samadi MT, Shakerkhatibi M, Poorolajal J, Rahmani A, Rafieemehr H, Hesam M. Association of long term exposure to outdoor volatile organic compounds (BTXS) with pro-inflammatory biomarkers and hematologic parameters in urban adults: A cross-sectional study in Tabriz, Iran. Ecotox Environ Safe. 2019;180:152-159.

27. Pelallo-Martínez NA, Batres-Esquivel L, Carrizales-Yáñez L, Díaz-Barriga FM. Genotoxic and Hematological Effects in Children Exposed to a Chemical Mixture in a Petrochemical Area in Mexico. Arch Environ Con Tox. 2014;67(1):1-8.

28. Hennig B, Ormsbee L, McClain CJ, Watkins BA, Blumberg B, Bachas LG, et al. Nutrition Can Modulate the Toxicity of Environmental Pollutants: Implications in Risk Assessment and Human Health. Environ Health Persp. 2012;120(6):771-774.

29. Sung J, Ho C, Wang Y. Preventive mechanism of bioactive dietary foods on obesity-related inflammation and diseases. Food Funct. 2018;9:6081-6095.

30. Kjøllesdal MKR, Smith GD, Ariansen I, Kinge JM, Degerud E, Næss $\emptyset$. The association between BMI and mortality using early adulthood BMI as an instrumental variable for midlife BMI. Sci Rep-UK. 2018;8(1):1-9.

31. Kupusinac A, Stokić E, Sukić E, Rankov O, Katić A. What kind of Relationship is Between Body Mass Index and Body Fat Percentage? J Med Syst. 2017;41(1):2-7. 
32. Guiraudou M, Varlet-Marie E, Raynaud De Mauverger E, Brun J. Obesity-related increase in whole blood viscosity includes different profiles according to fat localization. Clin Hemorheol Micro. 2013;55(1):63-73.

33. Vuong J, Qiu Y, La M, Clarke G, Swinkels DW, Cembrowski G. Reference intervals of complete blood count constituents are highly correlated to waist circumference: Should obese patients have their own "normal values?". Am J Hematol. 2014;89(7):671-677.

34. Brahmi N, Saoudi M, Kadri Y, Kallel C, Euch AE, Ayadi FM, et al. Protective effect of Chaetomorpha gracilis aqueous extract against erythrocytes oxidative damage induced by high fat diet in treated mice. Arch Physiol Biochem. 2019;125(3):220-227.

35. Valencak TG, Osterrieder A, Schulz TJ. Sex matters: The effects of biological sex on adipose tissue biology and energy metabolism. Redox Biol. 2017;12:806-813.

36. Moro AM, Brucker N, Charão MF, Baierle M, Sauer E, Goethel G, et al. Biomonitoring of gasoline station attendants exposed to benzene: Effect of gender. Mutat Res-Gen Tox En. 2017;813:1-9.

37. Wannamethee SG, Lowe GDO, Shaper AG, Rumley A, Lennon L, Whincup PH. Associations between cigarette smoking, pipe/cigar smoking, and smoking cessation, and haemostatic and inflammatory markers for cardiovascular disease. Eur Heart J. 2005;26(17):1765-1773.

38. Whitehead TP, Robinson D, Allaway SL, Hale AC. The effects of cigarette smoking and alcohol consumption on blood haemoglobin, erythrocytes and leucocytes: a dose related study on male subjects. Clin Lab Haematol. 1995;17(2):131-138.

39. Dobner J, Kaser S. Body mass index and the risk of infection - from underweight to obesity. Clin Microbiol Infec. 2018;24(1):24-28.

40. Sato A, Nakajima T, Fujiwara Y, Murayama N. Kinetic Studies on Sex Difference in Susceptibility to Chronic Benzene Intoxication: With Special Reference to Body Fat Content. Br J Ind Med. 1975;32(4):321-328.

41. Travis CC, Quillen JL, Arms AD. Pharmacokinetics of benzene. Toxicol Appl Pharm. 1990;102(3):400420.

42. Badham HJ, Winn LM. In utero and in vitro effects of benzene and its metabolites on erythroid differentiation and the role of reactive oxygen species. Toxicol Appl Pharm. 2010;244(3):273-279.

43. Unruh D, Srinivasan R, Benson T, Haigh S, Coyle D, Batra N, et al. Red Blood Cell Dysfunction Induced by High-Fat Diet. Circulation. 2015;132(20):1898-1908.

44. Yu J, Choi W, Lee H, Lee J. Relationship between inflammatory markers and visceral obesity in obese and overweight Korean adults. Medicine. 2019;98(9):e14740.

45. Prince OD, Langdon JM, Layman AJ, Prince IC, Sabogal M, Mak HH, et al. Late stage erythroid precursor production is impaired in mice with chronic inflammation. Haematologica. 2012;97(11):1648-1656.

46. Scharnagl H, Kleber ME, Genser B, Kickmaier S, Renner W, Weihrauch G, et al. Association of myeloperoxidase with total and cardiovascular mortality in individuals undergoing coronary angiography--the LURIC study. Int J Cardiol. 2014;174(1):96-105. 
47. Martinez-Hernandez A, Cordova EJ, Rosillo-Salazar O, Garcia-Ortiz H, Contreras-Cubas C, IslasAndrade S, et al. Association of HMOX1 and NQ01 polymorphisms with metabolic syndrome components. PLoS One. 2015;10(5):e0123313.

48. Wan J, Shi J, Hui L, Wu D, Jin X, Zhao N, et al. Association of genetic polymorphisms in CYP2E1, MPO, NQ01, GSTM1, and GSTT1 genes with benzene poisoning. Environ Health Perspect. 2002;110(12):1213-1218.

\section{Tables}

Due to technical limitations the tables are included in the Supplementary Files as a PDF download.

\section{Additional File Legends}

\section{Additional file 1. doc}

Table S1: Associations of urinary SPMA with erythroid-related hematologic parameters in different groups of gender, smoking status and drinking status; Table S2: Associations of BF\% with erythroidrelated hematologic parameters in different groups of smoking status and drinking status; Table S3: Associations of plasma TC levels with erythroid-related hematologic parameters in different groups of gender, smoking status and drinking status; Table S4: Associations of plasma TG levels with erythroidrelated hematologic parameters in different groups of gender, smoking status and drinking status; Table S5: Associations of fatty liver occurrence with erythroid-related hematologic parameters in different groups of gender, smoking status and drinking status; Table S6: Associations of urinary SPMA with erythroid-related hematologic parameters in different fat content categories.

\section{Figures}



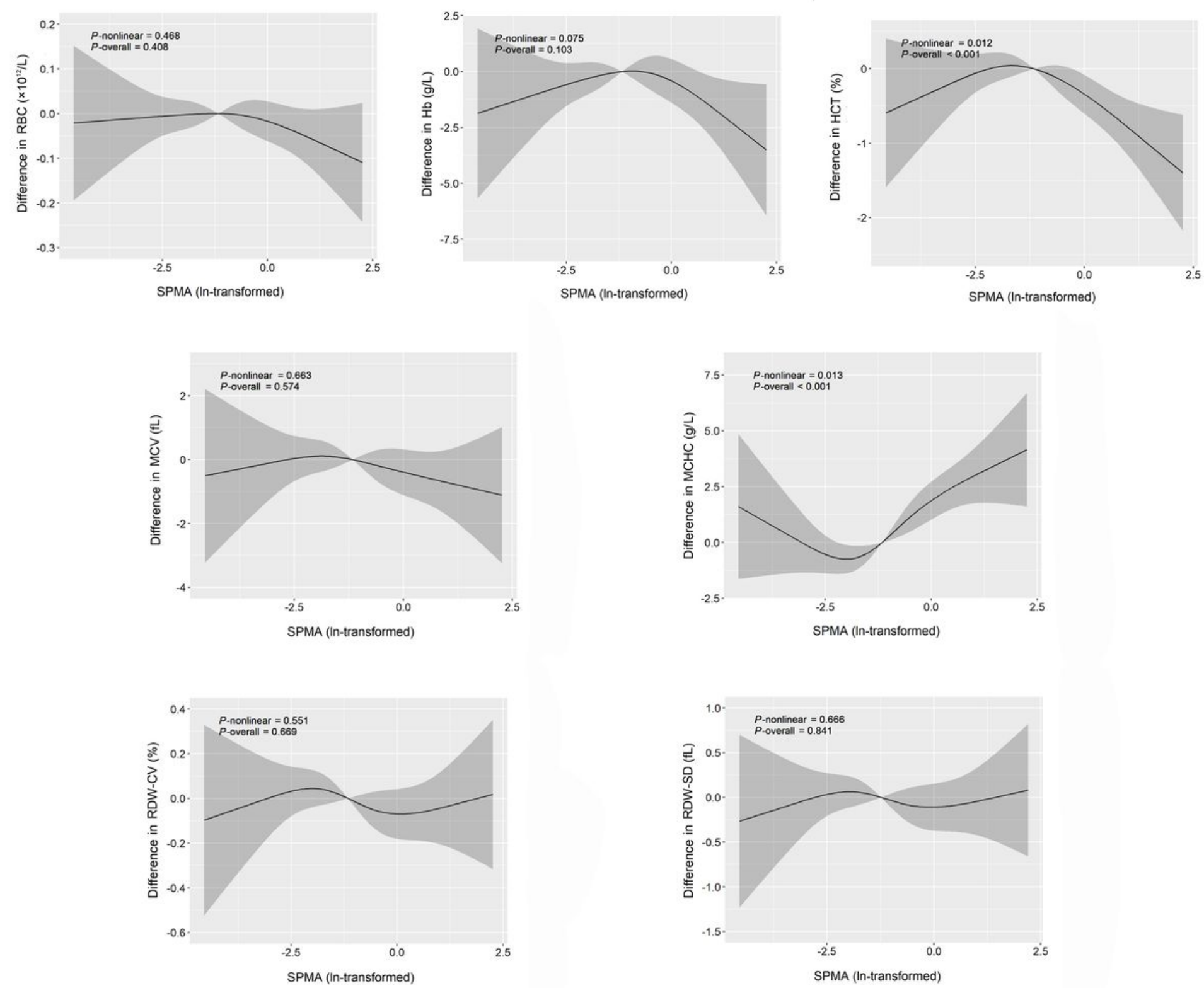

\section{Figure 1}

Adjusted dose-response associations of SPMA with erythroid-related hematologic parameters. 

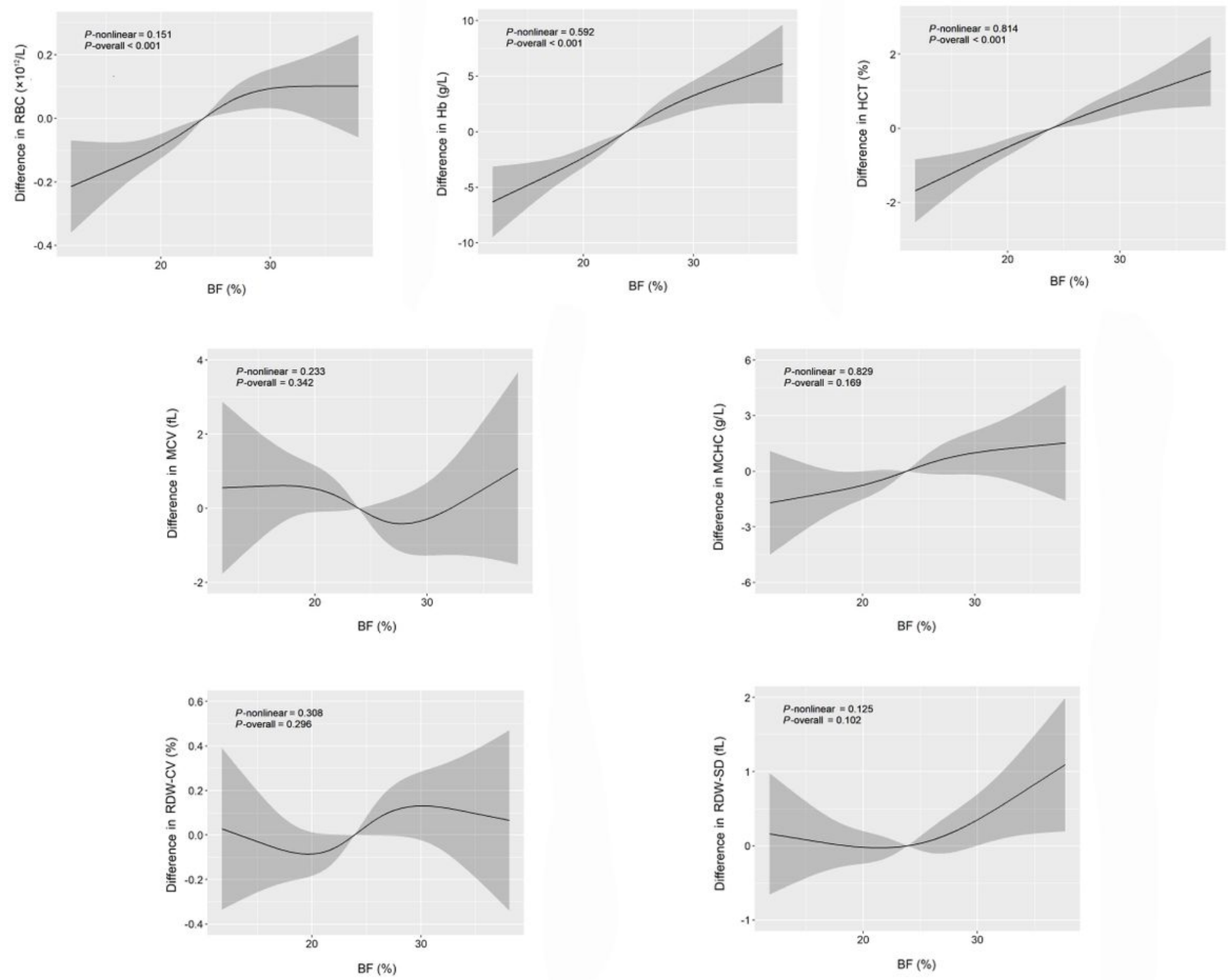

Figure 2

Adjusted dose-response associations of BF\% with erythroid-related hematologic parameters. 

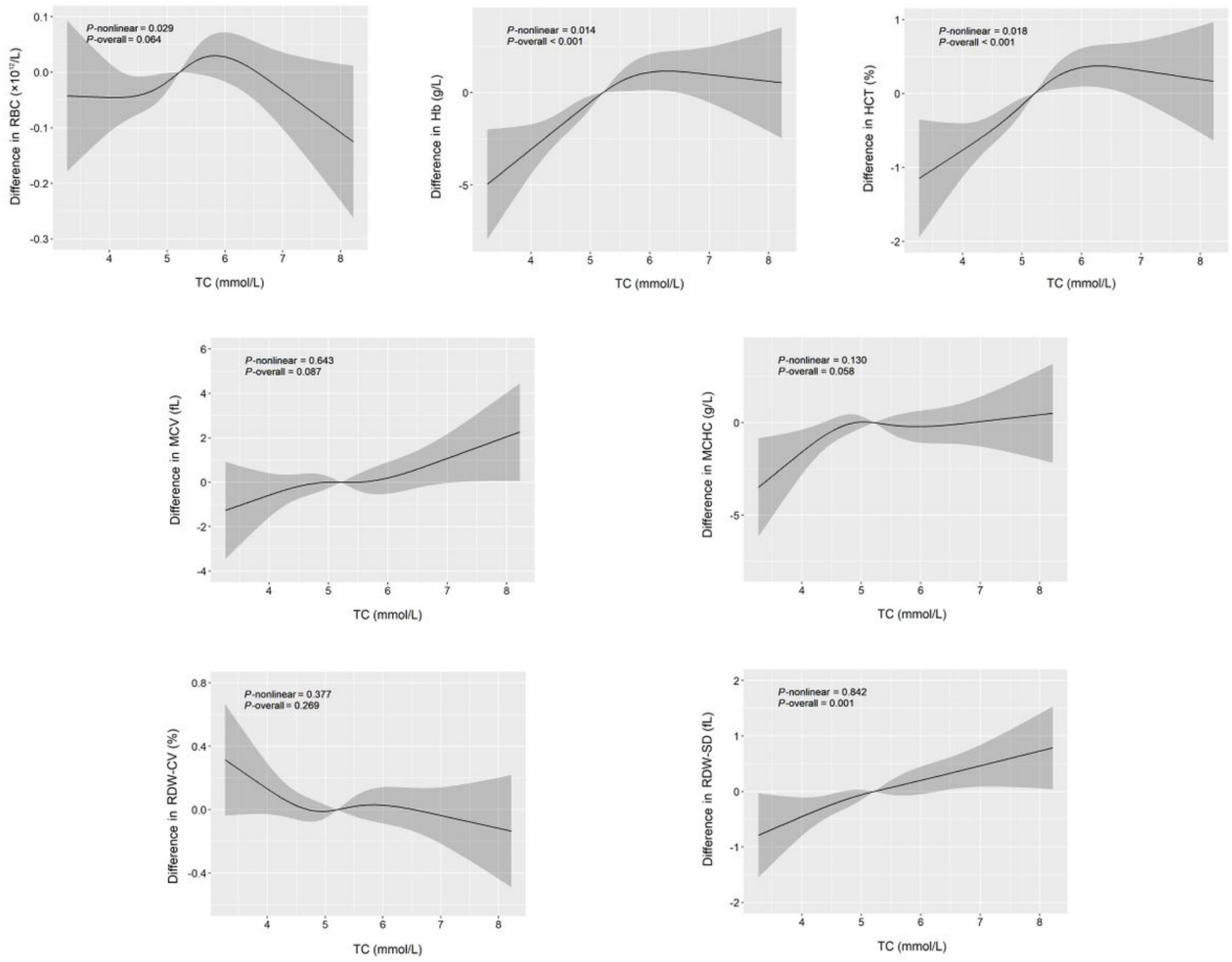

\section{Figure 3}

Adjusted dose-response associations of TC with erythroid-related hematologic parameters. 

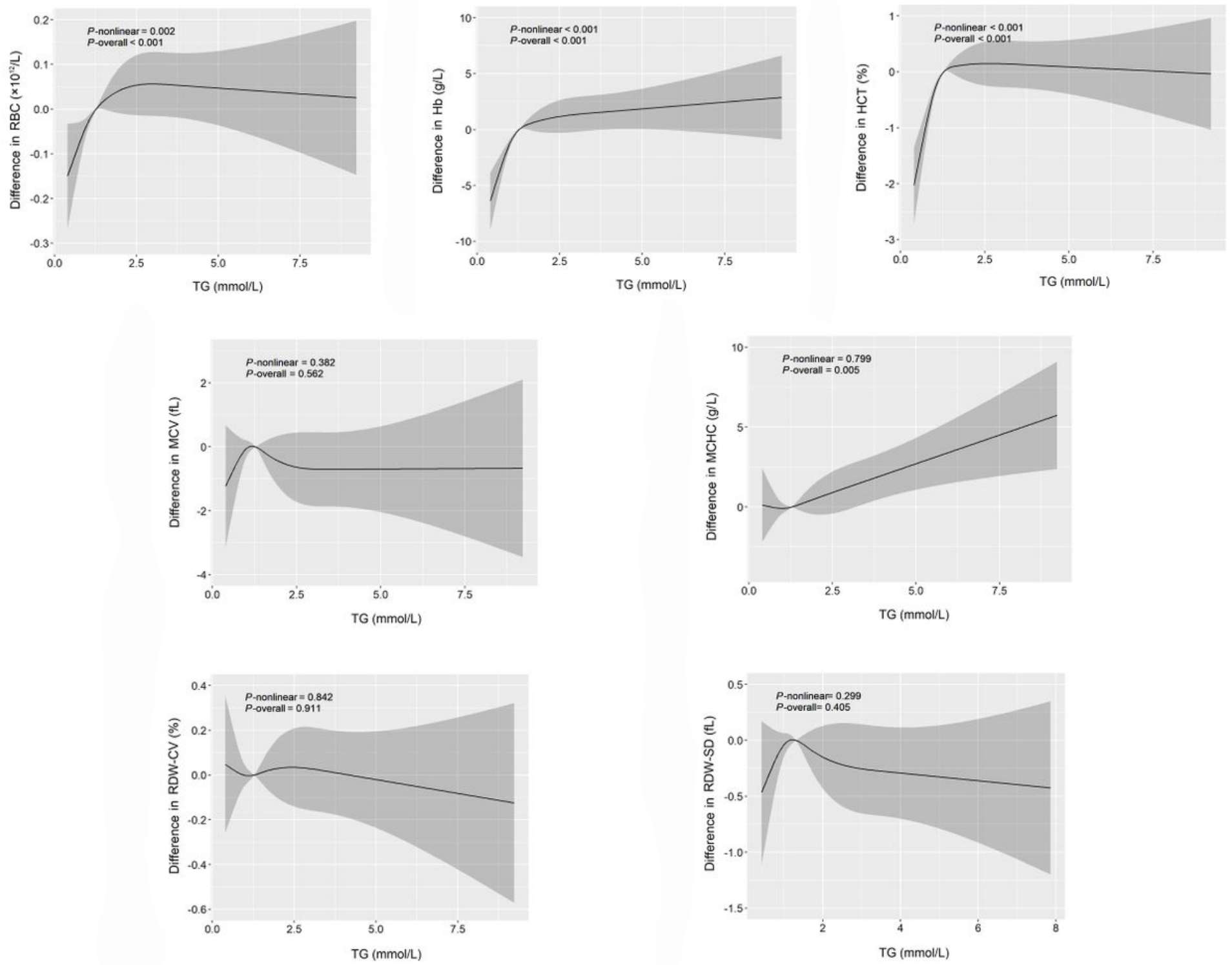

\section{Figure 4}

Adjusted dose-response associations of TG with erythroid-related hematologic parameters. 

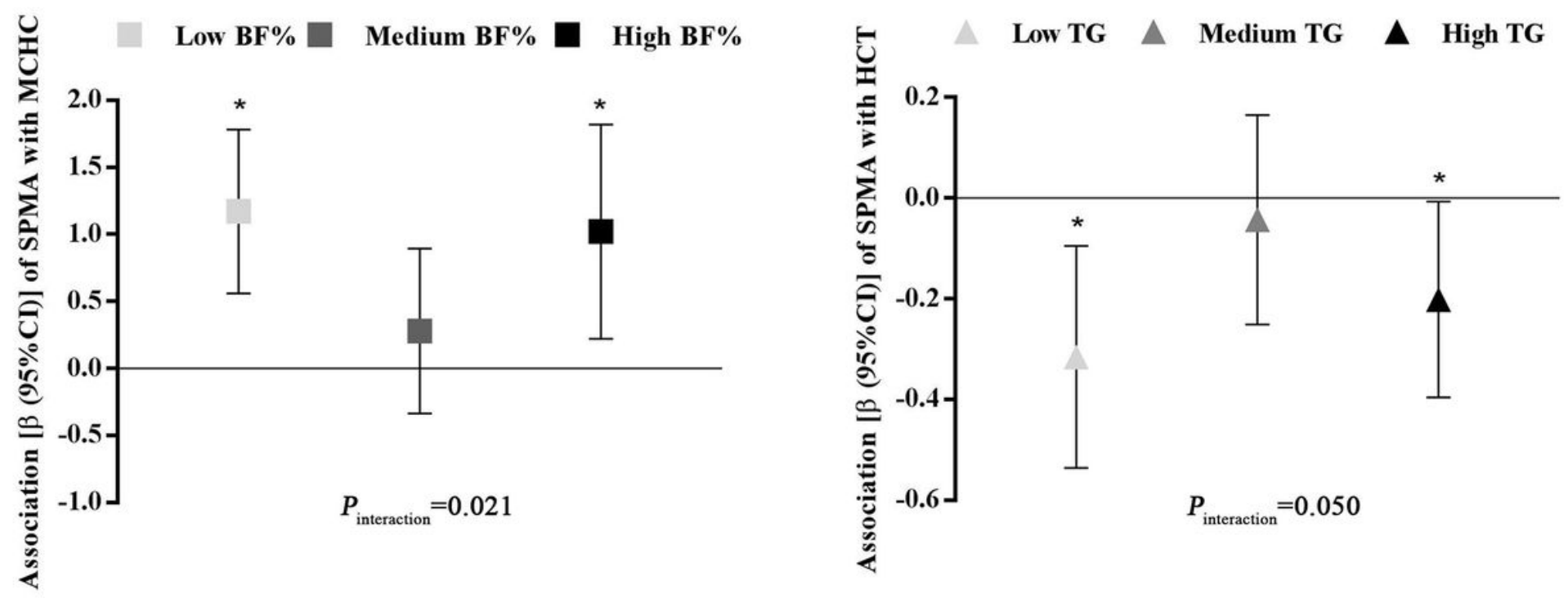

\section{Figure 5}

Significant modification effects of fat content on the associations between SPMA and erythroid-related hematologic parameters. The lines represent the associations $(\beta, 95 \% \mathrm{Cl})$, and $\mathrm{P}$ values for interaction effects are calculated by modeling an interaction term of SPMA and fat content in GLMs with adjustment for age, gender, smoking status, drinking status, work years, and workplace. * Ptrend $<0.05$, by GLMs adjusted for age, gender, smoking status, drinking status, work years, and workplace.

\section{Supplementary Files}

This is a list of supplementary files associated with this preprint. Click to download.

- Tables.pdf

- Additionalfile1.jpg

- Additionalfile2.doc 